\title{
Serum C1q concentration is associated with disease activity in Chinese Takayasu arteritis patients: a case-control study
}

\author{
Si Chen ${ }^{1}$, Haixia Luan ${ }^{1}$, Jianxun $\mathrm{He}^{1}$, Yan Wang ${ }^{1}$, Xiaoli Zeng ${ }^{1}$, Yongzhe $\mathrm{Li}^{1}$, and Hui \\ Yuan $^{1}$ \\ ${ }^{1}$ Affiliation not available
}

September 11, 2020

\begin{abstract}
Background: C1q is a crucial component of the classical complement pathway. This study is the first to assess the association between disease activity and serum levels of C1q in Chinese Takayasu arteritis (TA) patients. Methods: Serum C1q levels in $190 \mathrm{TA}$ patients and 154 healthy controls were assessed, and the relationship between serum C1q levels and indices of TA disease activity was analyzed. Moreover, we examined the correlation between serum C1q levels and two traditional inflammatory biomarkers; erythrocyte sedimentation rate (ESR) and hypersensitive CRP (hs-CRP). Results: Serum C1q levels were increased in TA patients compared with healthy controls $(\mathrm{p}=0.008)$. TA patients with active disease had higher levels of serum C1q than patients who had inactive disease $(\mathrm{p}<0.0001)$. Additionally, treatment-naïve patients had higher serum C1q levels than those who had been treated with corticosteroids or at least one immunosuppressant $(p=0.001)$. Furthermore, a positive correlation between serum C1q levels and traditional inflammatory biomarkers in TA patients was found. The role of $\mathrm{C} 1 \mathrm{q}$ in assessing disease activity was studied, and the area under the receiver operating characteristic (ROC) curve (AUC) of C1q for predicting active disease was 0.752 , and a serum cutoff value of $167.15 \mathrm{mg} / \mathrm{LC} 1 \mathrm{q}$ maximized the ability of disease activity assessment, with a sensitivity/specificity of $77.80 \% / 64.90 \%$. When the three indicators (C1q, ESR and hs-CRP) were combined, the AUC increased to 0.845 , and the sensitivity to $84.40 \%$. Conclusions: Serum C1q concentration may be a useful inflammatory marker for TA and a potential factor for evaluating disease activity in TA patients.
\end{abstract}

\section{INTRODUCTION}

Takayasu arteritis (TA) is an uncommon systemic vasculitis that is characterized by granulomatous inflammation of major blood vessels and primarily involves the aorta and its major branches. Vascular inflammation often leads to ischemia of organs and tissues supplied by the involved vessels and can result in potentially life-threatening organ ischemia as well as aortic regurgitation and pulselessness. ${ }^{1}$ The active stage of TA is associated with the development of ischemic symptoms such as coronary artery disease, stroke, and vision loss. ${ }^{2}$ Therefore, reliable disease activity assessment is important for preventing TA progression and endorgan ischemic injury. The erythrocyte sedimentation rate (ESR) and serum C-reactive protein (CRP) are the biomarkers most commonly used to monitor TA disease activity; however, as they may be influenced by several factors, they are neither sensitive nor specific. In clinical practice, some patients may undergo deterioration of vasculitis without elevation of CRP or ESR, and increases in CRP or ESR are found in only approximately half of patients with activeTA. ${ }^{3}$ Consequently, CRP and ESR do not always show a positive association with TA disease activity or severe vasculature impairment. ${ }^{4}$

Complement 1q $(\mathrm{C} 1 \mathrm{q})$ is an important promoter in the classical complement pathway. C1q initiates and activates the complement cascade by recognizing the complement-binding site of the antibody FC segment in the IgG or IgM immune complex to clear antigen-antibody complexes. ${ }^{5} \mathrm{C} 1 \mathrm{q}$, which accounts for approximately $70 \%$ of the $\mathrm{C} 1$ complex, can be deposited on the surface of apoptotic cells, facilitating phagocytosis by phagocytes and protecting the body from the inflammatory reactions. ${ }^{6}$ Serum C1q levels have recently 
been evaluated in several autoimmune, such as lupus nephritis (LN), ${ }^{7}$ pediatric systemic lupus erythematosus (PSLE), ${ }^{8}$ juvenile idiopathic arthritis $(\mathrm{JIA})^{9}$ and idiopathic inflammatory myopathies (IIMs). ${ }^{10}$ For example, Tan et al. found that the serum C1q level was markedly reduced in LN patients compared with normal controls, revealing a correlation with LN disease activity and renal total activity index scores. ${ }^{7}$ In addition, Wu et al. showed that the level of serum C1q in PSLE patients was significantly lower than that in healthy children as well as in children with other rheumatic diseases and that the level of serum C1q correlated negatively with the disease active index. ${ }^{8}$ Furthermore, Gilliam et al. reported that in JIA patients, mean serum levels of C1q were significantly increased compared with those in healthy children, ${ }^{9}$ and Li et al. indicated significant increases in serum C1q levels in a PM/DM patient group with elevated ESR compared with a group with normal ESR. ${ }^{10}$ However, the serum C1q level and its association with disease activity have not been investigated in TA patients. This study is the first to examine serum C1q levels in Chinese TA patients and investigate their role in the assessment of TA disease activity.

\section{MATERIALS AND METHODS}

\subsection{Study population}

We designed a retrospective study recruiting 190 subjects with TA diagnosed according to the criteria of the American College of Rheumatology (ACR). ${ }^{11}$ All TA patients were screened in Beijing Anzhen Hospital between September 2015 and August 2019. Patients who had other autoimmune diseases were excluded.154 healthy unrelated age- and sex-matched controls without any history of chronic disease were recruited during their physical examinations. Disease activity was assessed in patients with TA based on the National Institutes of Health (NIH) criteria proposed by Kerr et al. ${ }^{12}$ Clinical classification of TA (I, IIa, IIb, III, IV and $\mathrm{V}$ ) patients was according to the Numano criteria by catheterography or computed tomography angiography (CTA). ${ }^{13,14}$ Written informed consent was obtained from all study participants. The study was approved by the Ethical Committee of Beijing Anzhen Hospital, Capital Medical University.

\subsection{Measurement of serum C1q level}

Serum C1q levels were determined with an automatic biochemical analyzer (AU5400, Beckman, US) and C1q Reagent Kit (Beijia Biochemistry Reagents Co., Ltd, Shanghai, China). At the same time, blood white cell counts, biochemical parameters, ESR and hs-CRP levels were measured. All tests were performed according to the manufacturer's manual.

\subsection{Statistical analysis}

All statistical analyses were conducted with SPSS version 23.0 (SPSS Inc., Chicago, Illinois, USA) software. Numerical data were compared with an independent sample $t$-test or the Mann-Whitney-Wilcoxon test. Categorical data were compared with the Chi-square test or Fisher's exact test. Spearman's nonparametric correlation test was applied to examine the associations between serum C1q levels and Kerr's score/ESR/hsCRP. We selected the cutoff values for serum C1q, ESR, hs-CRP and the combination of three indicators (C1q, ESR and hs-CRP) using receiver operating characteristic (ROC) curves with MedCalc software (v.15.2) to compare the accuracies of these markers with disease activity identification. The cutoff points of these markers were the values with the highest Youden's Index (sensitivity+specificity-1) score. A $p$-value less than 0.05 was considered to be statistically significant.

\section{RESULTS}

Of the 190 TA patients, 178 were female, and 47patients had active disease based on the NIH criteria (Table 1). In addition, 29 of the TA patients in our study were naïve to corticosteroid or immunosuppressant treatment. Malaise (71.1\%), headache (47.9\%) and chest distress (27.4\%) were the three most common constitutional symptoms, and Numano subtype $\mathrm{V}$ was common among the TA patients. The prevalence of claudication differed between the TA patients with active and stable disease $(p=0.018)$. Four of the patients with active disease had Numano subtype IIa, whereas no Numano IIa cases were in clinical remission $(p=0.003)$, Furthermore, the prevalence of hypertension was notable between untreated TA patients and treated patients $(p=0.003)$. 
Compared with the healthy controls, patients with TA had higher ESR and C1q levels (Table 1, Figure 1). However, the level of hs-CRP was similar between the TA patients and healthy controls. Compared with patients who had inactive disease, those with active disease had higher levels of serum C1q and hs-CRP as well as ESR (Table 1, Figure 1). Similarly, treatment-naïve patients had higher serumC1q, ESR and hs-CRP than those who had always been treated with corticosteroids or at least one immunosuppressant (Table 1, Figure 1). We further analyzed the relationship between serum C1qand Kerr's score/ESR/hs-CRP with the Spearman correlation test and found that in our TA patients, serum C1q levels correlated significantly with Kerr's score, ESR and hs-CRP (Table 2).

The areas under the ROC curve (AUCs) for C1q, ESR and hs-CRP were 0.752, 0.825, and 0.834, respectively, though without significant differences (Table 3, Figure 2). Nevertheless, when the three indicators (C1q, ESR and hs-CRP) were combined, the AUC increased to 0.845. At the same time, the AUCs between the combination of the three indicators (C1q, ESR and hs-CRP) and hs-CRP with C1q were significantly different from each other. A serum cutoff value of $167.15 \mathrm{mg} / \mathrm{L}$ C1q maximized the disease activity assessment capacity, with a sensitivity/specificity of $77.80 \% / 64.90 \%$. Using the established ESR and hs-CRP thresholds of our center, ESR and hs-CRP were able to identify disease activity with a sensitivity/specificity of $80.00 \% / 81.70 \%$ and $70.20 \% / 86.50 \%$, respectively, and the sensitivity increased to $85.10 \%$ when the three indicators (C1q, ESR and hs-CRP) were combined (Table 3, Figure 2).

\section{DISCUSSION}

This study first analyzed the association of serum C1q levels with disease activity in a large sample of Chinese TA patients. Our experimental results showed that serum C1q levels were increased in TA patients compared to healthy controls. Notably, TA patients with active disease had higher levels of serum C1q than patients who had inactive disease. Serum C1q levels and traditional inflammatory biomarkers in TA patients correlated positively; therefore, our findings show that serum C1q concentrations is a potential biomarker for evaluating disease activity in TA patients. TA is a systemic autoimmune vasculitis with unknown etiology. Immune mechanisms appear to be involved in TA pathogenesis, as inflammatory cell infiltration along with granulomatous inflammation and excessive proinflammatory cytokine productions are observed in the vascular tissue involved. ${ }^{15}$ The pathogenesis of systemic vasculitides, including TA, correlates with the presence of inflammatory cells in the arteries and with immune complex (IC) deposition, which cause activation of the complement system, followed by the inflammation and destruction of vascular mural structures. ${ }^{16}$ Previous studies have found ICs in TA patient sera and on peripheral blood lymphocyte Fc receptors. ${ }^{17}$ Additionally, a recent paper analyzed the proteomics of circulating ICs in the sera of TA patients and identified three unique antigens. ${ }^{18}$ Although there are some studies on the relationship between IC and TA, analyses of the association between the complement system and TA have not been reported to date. Moreover, whether activation of the complement system is involved in the pathogenesis of TA remains unclear. $\mathrm{C} 1 \mathrm{q}$, historically viewed as the initiating component of the classical complement pathway, has a diverse range of functions in both innate and acquired immunity. Indeed, C1q plays important roles in the pathogenesis of autoimmune diseases, particularly systemic lupus erythematosus (SLE) ${ }^{19}$ However, the relationship between C1q and TA remains unresolved. This study is the first to investigate the serum C1q level in Chinese TA patients and its role in the assessment of TA disease activity.

Several studies have suggested the use of serum C1q as an inflammatory marker in multiple diseases. For example, the C1q level in serum is considered an inflammatory marker for disease activity in childhood $\mathrm{LN}^{7}$ and PM/DM. ${ }^{10}$ Our study found that TA patients with active disease had higher levels of serum C1q than patients who had inactive disease. At the same time, treatment-naïve patients had higher serum C1q levels than those who had always been treated with corticosteroids or at least one immunosuppressant. We found serum C1q levels to correlate significantly with Kerr's score, ESR and hs-CRP levels in our TA patients. The AUC ofC1qwas lower than that of ESR, but the AUC of C1q was higher than that of hs-CRP. In addition, when the three indicators (C1q, ESR and hs-CRP) were combined, the AUC increased, and a serum cutoff value of $167.15 \mathrm{mg} / \mathrm{L} \mathrm{C1q} \mathrm{maximized} \mathrm{the} \mathrm{disease} \mathrm{activity} \mathrm{assessment} \mathrm{capacity.} \mathrm{The} \mathrm{sensitivity}$ of C1q was lower than that of ESR and higher than that of hs-CRP, though the specificity of C1q was 
lower than that of both ESR and hs-CRP. Additionally, when the three indicators (C1q, ESR and hs-CRP) were combined, the sensitivity increased. Therefore, hs-CRP has the highest specificity, and the sensitivity of combining three indicators (C1q, ESR and hs-CRP) was the highest. Based on the above, we conclude that the concentration of serum C1qisa potential inflammatory marker for TA and that the combination of the above three indicators increases the sensitivity of disease activity assessment.

A limitation of this study is that we did not detect $\mathrm{C} 1 \mathrm{q}$ deposited in vascular tissue. As a result, well-designed prospective studies should be performed to clarify the exact clinical molecular mechanisms of C1q in TA patients and the association with TA disease activity.

\section{CONCLUSION}

The present study is the first to assess serum C1q levels in TA patients and evaluate their relationship with disease activity. We found that serum C1q levels were increased in TA patients compared to healthy controls and that TA patients with active disease had higher levels of serum C1q than patients who had inactive disease. Additionally, there were positive correlations between serum C1q levels and traditional inflammatory biomarkers in TA patients. Therefore, our findings show that the serum C1q concentration is a potential inflammatory marker for TA and that the combination of three indicators (C1q, ESR and hs-CRP) increases the sensitivity of disease activity assessment.

\section{ACKNOWLEDGEMENTS}

This work was supported by the National Natural Science Foundation of China Grants (No. 81800435), by the Youth Plan of Beijing Hospital Management Center (QML20190602), by the Beijing Excellent Talents Training Support (2018000021469G242).

\section{CONFLICT OF INTERESTS}

None declared.

\section{REFERENCE}

1. de Souza A W, de Carvalho J F. Diagnostic and classification criteria of Takayasu arteritis. J Autoimmun . 2014;48-49(79-83).

2. Grayson P C, Cuthbertson D, Carette S, et al. New features of disease after diagnosis in 6 forms of systemic vasculitis. J Rheumatol . 2013;40(11):1905-1912.

3. Tombetti E, Di Chio M C, Sartorelli S, et al. Systemic pentraxin-3 levels reflect vascular enhancement and progression in Takayasu arteritis. Arthritis Res Ther . 2014;16(6):479.

4. Dogan S, Piskin O, Solmaz D, et al. Markers of endothelial damage and repair in Takayasu arteritis: are they associated with disease activity? Rheumatol Int . 2014;34(8):1129-1138.

5. Sjoberg A P, Trouw L A, Blom A M. Complement activation and inhibition: a delicate balance. Trends Immunol . 2009;30(2):83-90.

6. Castellano G, Woltman A M, Nauta A J, et al. Maturation of dendritic cells abrogates C1q production in vivo and in vitro. Blood . 2004;103(10):3813-3820.

7. Tan Y, Song D, Wu L H, et al. Serum levels and renal deposition of C1q complement component and its antibodies reflect disease activity of lupus nephritis. BMC Nephrol . 2013;14(63.

8. Wu F Q, Zhao Q, Cui X D, et al. C1q and anti-C1q antibody levels are correlated with disease severity in Chinese pediatric systemic lupus erythematosus. Rheumatol Int . 2011;31(4):501-505.

9. Gilliam B E, Reed M R, Chauhan A K, et al. Significance of complement components C1q and C4 bound to circulating immune complexes in juvenile idiopathic arthritis: support for classical complement pathway activation. Clin Exp Rheumatol . 2011;29(6):1049-1056. 
10. Li L, Chen J, Chen S, et al. Serum C1q Concentration Positively Correlates with Erythrocyte Sedimentation Rate in Polymyositis/Dermatomyositis.Ann Clin Lab Sci . 2019;49(2):237-241.

11. Arend W P, Michel B A, Bloch D A, et al. The American College of Rheumatology 1990 criteria for the classification of Takayasu arteritis. Arthritis Rheum . 1990;33(8):1129-1134.

12. Kerr G S, Hallahan C W, Giordano J, et al. Takayasu arteritis. Ann Intern Med . 1994;120(11):919-929.

13. Ueno A, Awane Y, Wakabayashi A, et al. Successfully operated obliterative brachiocephalic arteritis (Takayasu) associated with the elongated coarctation. Jpn Heart J . 1967;8(5):538-544.

14. Lupi E, Sanchez G, Horwitz S, et al. Pulmonary artery involvement in Takayasu's arteritis. Chest . 1975;67(1):69-74.

15. Vaideeswar P, Deshpande J R. Pathology of Takayasu arteritis: A brief review. Ann Pediatr Cardiol . 2013;6(1):52-58.

16. Chimenti M S, Ballanti E, Triggianese P, et al. Vasculitides and the Complement System: a Comprehensive Review. Clin Rev Allergy Immunol . 2015;49(3):333-346.

17. Gyotoku Y, Kakiuchi T, Nonaka Y, et al. Immune complexes in Takayasu's arteritis. Clin Exp Immunol . 1981;45(2):246-252.

18. Ohyama K, Baba M, Tamai M, et al. Proteomic profiling of antigens in circulating immune complexes associated with each of seven autoimmune diseases. Clin Biochem . 2015;48(3):181-185.

19. Son M, Diamond B, Santiago-Schwarz F. Fundamental role of C1q in autoimmunity and inflammation. Immunol Res . 2015;63(1-3):101-106.

TABLE 1 Demographic, clinical characteristics and laboratory findings between TA patients and healthy controls

\begin{tabular}{|c|c|c|}
\hline & HC $($ Mean \pm SEM/ n)/ Median $(25 \%, 75 \% \mathrm{Q})$ & TA $($ Mean \pm SEM/ n)/ Median $(25 \%, 75 \% \mathrm{Q})$ \\
\hline Female & $136 / 154$ & $178 / 190$ \\
\hline Age (years) & $38.05 \pm 9.07$ & $36.03 \pm 12.70$ \\
\hline \multicolumn{3}{|c|}{ Constitutional symptoms } \\
\hline Fever & 1 & $8 / 190$ \\
\hline Malaise & 1 & $135 / 190$ \\
\hline Arthralgia/ Arthritis & 1 & $12 / 190$ \\
\hline Headache & / & $91 / 190$ \\
\hline Chest distress/pain & / & $52 / 190$ \\
\hline Carotidynia & / & $16 / 190$ \\
\hline \multicolumn{3}{|l|}{ Vascular findings } \\
\hline Claudication & / & $15 / 190$ \\
\hline Bruits & / & $127 / 190$ \\
\hline Pulsation weakened & / & $143 / 190$ \\
\hline Pulse deficit & / & $58 / 190$ \\
\hline Asymmetric BP & / & $104 / 190$ \\
\hline Hypertension & / & $90 / 190$ \\
\hline \multicolumn{3}{|l|}{ Laboratory data } \\
\hline $\operatorname{ALT}(\mathrm{U} / \mathrm{L})$ & $14.40(9.00-19.00)$ & $21.39(11.00-24.00)$ \\
\hline $\operatorname{Scr}(\mu \mathrm{mol} / \mathrm{L})$ & $59.88(52.50-68.20)$ & $54.62(46.75-61.28)$ \\
\hline $\mathrm{WBC}\left(10^{9} / \mathrm{L}\right)$ & $5.59(4.26-6.30)$ & $7.24(4.91-8.40)$ \\
\hline $\mathrm{Hb}(\mathrm{g} / \mathrm{L})$ & $133.99(125.00-138.00)$ & $123.30(114.75-133.00)$ \\
\hline $\operatorname{PLT}\left(10^{9} / \mathrm{L}\right)$ & $257.17(216.00-300.00)$ & $238.02(193.75-278.25)$ \\
\hline ESR (mm/hour) & $5.58(3.00-8.00)$ & $10.00(3.00-12.00)$ \\
\hline
\end{tabular}




\begin{tabular}{|c|c|c|}
\hline & HC $($ Mean \pm SEM/ n)/ Median $(25 \%, 75 \% \mathrm{Q})$ & $\mathrm{TA}($ Mean $\pm \mathrm{SEM} / \mathrm{n}) /$ Median $(25 \%, 75 \% \mathrm{Q})$ \\
\hline hs-CRP (mg/L) & $0.90(0.37-1.03)$ & $3.74(0.13-2.29)$ \\
\hline $\mathrm{C} 1 \mathrm{q}(\mathrm{mg} / \mathrm{L})$ & $150.11(143.73-158.30)$ & $166.85(140.70-185.93)$ \\
\hline \multicolumn{3}{|l|}{ Numano subtypes } \\
\hline I & / & $9 / 190$ \\
\hline IIa & / & $4 / 190$ \\
\hline IIb & / & $36 / 190$ \\
\hline III & / & $7 / 190$ \\
\hline IV & / & $17 / 190$ \\
\hline V & / & $117 / 190$ \\
\hline
\end{tabular}

HC: Healthy Controls; TA: Takayasu arteritis; SEM: standard error of mean; BP: blood pressure; ALT: alanine aminotransferase; Scr: serum creatinine; WBC: white blood cell; Hb: haemoglobulin; PLT: platelet; ESR: erythrocyte sedimentation rate; hs-CRP: hypersensitive C-reactive protein.

TABLE 2 Correlation of C1q with disease activity in patients with TA

\begin{tabular}{|c|c|c|c|c|c|c|}
\hline & Kerr' s score & Kerr' s score & hs-CRP & hs-CRP & ESR & ESR \\
\hline & $\mathrm{r}$ & $\mathrm{p}$ & $\mathrm{r}$ & $\mathrm{p}$ & & $\mathrm{p}$ \\
\hline $\mathrm{C} 1 \mathrm{q}$ & 0.460 & $<0.0001$ & 0.591 & $<0.0001$ & 0.604 & $<0.0001$ \\
\hline
\end{tabular}

ESR: erythrocyte sedimentation rate; hs-CRP: hypersensitive C-reactive protein.

TABLE 3 The sensitivity and specificity of serum C1q and other indicators to assess TA disease activity.

\begin{tabular}{llllll}
\hline & AUC & Sensitivity (\%) & Specificity (\%) & $95 \%$ CI & P \\
\hline C1q & 0.752 & 77.8 & 64.9 & $0.677-0.828$ & $<0.0001$ \\
hs-CRP & 0.834 & 70.2 & 86.5 & $0.770-0.898$ & $<0.0001$ \\
ESR & 0.825 & 80.0 & 81.7 & $0.748-0.901$ & $<0.0001$ \\
C1q+ hs-CRP+ESR & 0.845 & 85.1 & 77.3 & $0.775-0.915$ & $<0.0001$ \\
\hline
\end{tabular}

ESR: erythrocyte sedimentation rate; hs-CRP: hypersensitive C-reactive protein; AUC: area under the ROC curve; CI: confidence interval.

\section{Figure Legends}

FIGURE 1 A: Serum C1q levels were significantly higher in TA patients than in healthy controls $(p=0.008)$; B: Serum C1q levels were significantly higher in TA patients with active disease than inpatients who had inactive disease $(p<0.0001)$; C: Serum C1q levels were significantly higher in treatment-naïve patients than in those who had always been treated with corticosteroids or at least one immunosuppressant $(p=0.001)$.

TA: Takayasu arteritis.

FIGURE 2 The ROC curves for Cq, ESR, hs-CRP and the combination of the three indicators in 190 TA patients according to NIH criteria.

ROC: receiver operating characteristics; AUC: area under the ROC curve; ESR: erythrocyte sedimentation rate; hs-CRP: hypersensitivity C-reactive protein; TA: Takayasu arteritis; NIH: National Institutes of Health. 

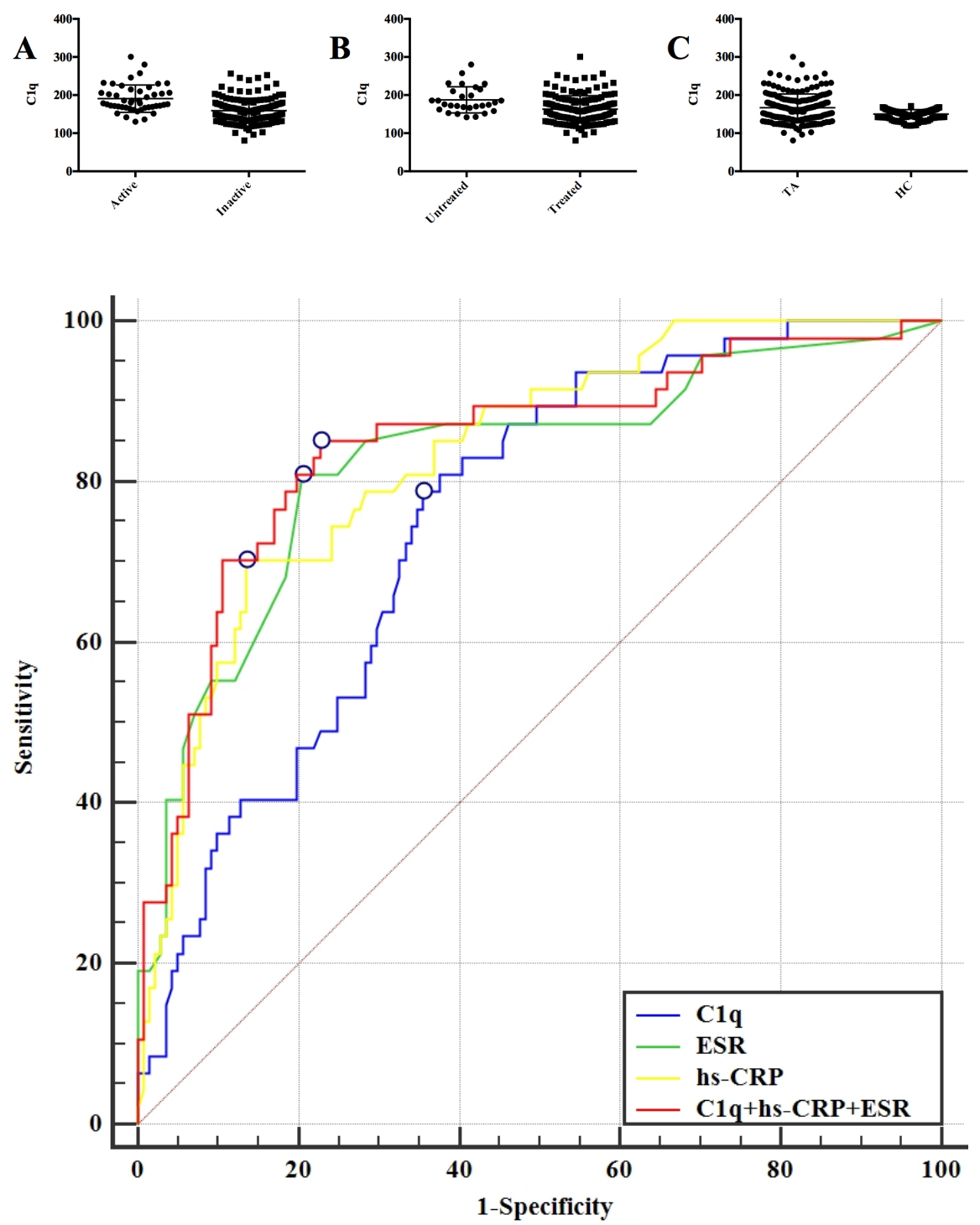